\title{
Los contenidos de los mensajes para la salud alimentaria en los libros de texto de la escuela primaria
}

\author{
Juan Manuel Muñoz Cano \\ Universidad Juárez Autónoma de Tabasco \\ (Villahermosa, México) \\ Teresita del Niño Jesús Maldonado Salazar \\ Centro de Capacitación para el Desarrollo Sustentable \\ (Ciudad de México D.F., México)
}

\section{Palabras clave}

educación primaria

libros de texto

análisis de contenidos

nutrición

estilo de vida

México

\begin{abstract}
Resumen
El objetivo de este estudio fue analizar las características técnicas y el sustento científico de los mensajes relacionados con la nutrición en los libros de los educandos del nivel de educación primaria de México. Durante la investigación se revisaron los contenidos en los libros de ciencias naturales y las sugerencias pedagógicas para los maestros desde la Secretaría de Educación así como las temáticas de los libros de apoyo de autores de varios países que se distribuyen a las bibliotecas de las escuelas. Como resultado se obtuvo que los mensajes no contienen los elementos para contrarrestar lo difundido en los medios que promueven alimentos y bebidas obesogénicos. Un número elevado de mensajes mencionan enfermedades consecuencia de la obesidad. Los datos que contienen los textos no consideran las investigaciones científicas y los saberes tradicionales: se centran en grupos de alimentos y las equivalencias. Se concluye que es necesario que los mensajes se promuevan de acuerdo a los nuevos paradigmas de la nutrición con base en alimentos específicos y los conocimientos ancestrales.
\end{abstract}

Este trabajo es parte del proyecto "Estrategia de intervención educativa para la salud alimentaria" financiado por Fondos Mixtos del Consejo Nacional de Ciencia y Tecnología-Gobierno de Tabasco, México, clave TAB2010-C19-144012.

Contacto para correspondencia: Juan Manuel Muñoz Cano. Email: juan.munoz@ujat.mx. Teléfono: +52 9933581500 extensión 6313 . 


\section{Nutritional Health Message Content in Primary School Textbooks}

\begin{tabular}{c}
\hline Keywords \\
\hline primary education \\
textbooks \\
content analysis \\
nutrition \\
lifestyle \\
Mexico
\end{tabular}

\section{Cómo citar el artículo}

Muñoz Cano, J. M. y Maldonado Salazar, T. (2013). Los contenidos de los mensajes para la salud alimentaria en los libros de texto de la escuela primaria. Revista de Comunicación y Salud, 3(1), pp. 19-33.

DOI: http://doi.org/10.35669/revistadecomunicacionysalud.2013.3(1).19-33 
La educación es comunicación, es diálogo, en la medida en que no es la transferencia del saber sino un encuentro de sujetos interlocutores.

Paulo Freire. Extensión y comunicación

\section{Introducción}

La nutrición es uno de los ejes del estilo de vida por lo que constituye un importante núcleo en la educación básica. Formar a los estudiantes de la escuela primaria para que seleccionen alimentos y bebidas saludables ha de ser primordial para el logro de las capacidades en la toma de decisiones sobre nutrición deseable. La construcción de estas capacidades depende tanto de un proceso de acompañamiento docente como de que la información que reciben los educandos tenga sustento científico. En México esta fuente son los libros de texto gratuito aunque también se distribuyen en las escuelas colecciones de libros para conformar bibliotecas escolares: Los libros del rincón. Esto tiene el fin de que tengan información adicional para la realización de sus proyectos escolares.

Los libros de texto gratuitos se distribuyen desde 1959 por la Secretaría de Educación en todas las escuelas del gobierno y de cuotas del país. Los libros han evolucionado a la par que las reformas en planes, programas y propuestas pedagógicas (Anzures, 2011). Aunque algunas escuelas cuentan con otros recursos e incluso con acceso a internet y software educativo, la mayor parte de las actividades se realizan con base en los libros de texto. En este marco es relevante analizar la información que se comunica a los escolares a través de los diversos libros para evaluar su pertinencia y, en su caso, las necesidades de reconfiguración.

\subsection{La promoción del estilo de vida saludable}

Es alto el número de mensajes emitidos en los medios a los que se encuentran expuestos los educandos, los maestros y los padres de familia. Sólo en México se estima que un niño promedio mira dos horas al día la televisión, donde existe "saturación de mensajes en medios de comunicación masiva orientados al consumo de alimentos con bajo o nulo valor nutrimental" (Gobierno Federal [GF, en adelante], 2010: 4). Esto ha influido de forma importante en los hábitos de consumo de alimentos y bebidas. Se han cambiado los alimentos tradicionales, típicos, por lo que se denomina dieta occidentalizada, moderna. Ésta se promueve como fácil, divertida, barata. Como consecuencia de ello, México tuvo en 2006 una prevalencia combinada de sobrepeso y obesidad del $26 \%$, que ha continuado en ascenso como resultado del consumo de los alimentos obesogénicos. De acuerdo con el diagnóstico realizado por el Gobierno de México, "es fundamental adoptar medidas que fortalezcan una adecuada educación para la salud que sea congruente con el tipo de alimentos al que tienen acceso los niños, niñas y adolescentes en el ambiente escolar" (GF, 2010: 7).

De esta manera consideramos que la estructura conceptual de la información científica requiere de análisis a la luz de los avances que emergen de estudios clínicos, epidemiológicos y 
experimentales que cuestionan la validez de las construcciones en las que se han basado los contenidos conceptuales acerca de la nutrición para el estilo de vida. Esas construcciones se plantearon antes de la pandemia de sobrepeso y obesidad por lo que es cuestionable su efectividad, toda vez que en su elaboración se partió de ideas generales acerca de macronutrimentos y calorías, y no se tenía claro el papel de los componentes de los alimentos en las modificaciones epigenéticas (encendido y apagado de genes) que pueden desencadenar enfermedades crónicas no transmisibles independientemente de que se tenga sobrepeso o no (Nakamura y Omalle, 2012). Así, en vez de ser herramientas útiles para la educación en una alimentación saludable, estos modelos contribuyen a la pandemia de enfermedades crónicas no transmisibles, entre ellas la obesidad (Willet y Ludwig, 2012).

Propuestas como "El plato del buen comer" de México [en adelante también "Plato"], "La nueva rueda de los alimentos" de España, y la más reciente, "MyPlate", de Estados Unidos (USDA, 2011), se basan en esa misma estructura de las equivalencias de acuerdo al contenido de macronutrimentos. En éstas se considera semejante a los cereales integrales y a las harinas refinadas. En la representación gráfica "Plato" se muestra que los equivalentes al pan de trigo integral pueden ser un waffle o un pan dulce, elaborados con harina de trigo refinada, que aportan fructosa y colesterol. Las citadas propuestas también consideran que son sustituibles las leguminosas por las carnes rojas (o los embutidos, como en la versión española), lo cual se contrapone a la evidencia científica que sustenta el consumo diario de las primeras y evitar las segundas.

Lo anterior denota la necesidad de la reconfiguración de la información de las propuestas para una alimentación saludable, que se podría plantear según las siguientes sugerencias:

1. Basarlas en estudios científicos. Tal es el caso de la recomendación del consumo de agua simple potable del Instituto Nacional de Salud Pública de México;

2. Tomar en cuenta información consistente fundada en los datos científicos;

3. Abrir debates científicos para que se aporten recomendaciones de acuerdo a las revisiones de los resultados;

4. Elaborar las recomendaciones al público de acuerdo a las recomendaciones de los comités científicos;

5. Basar las recomendaciones en alimentos, no nutrimentos;

6. Incluir en las recomendaciones explícitamente qué alimentos deben consumirse en menor cantidad ya que son obesogénicos, y su consumo por sí mismo es un riesgo para enfermedades crónicas no transmisibles (Willet y Ludwig, 2012);

7. Anteponer el bienestar de la población a los intereses económicos.

Los tres últimos puntos, sobre todo el 5 , se refieren a aspectos que son la base de una propuesta diferente, que toma en consideración saberes y prácticas culinarias ancestrales que, a su vez, se sustentan en los datos científicos. En México, después del reconocimiento de la cocina tradicional como Patrimonio Intangible de la Humanidad (UNESCO, 2010), se han adherido a su promoción como parte de una estrategia para disminuir el riesgo de las enfermedades crónicas no transmisibles tanto figuras de la cocina (De los Reyes, 2011) como científicos (UNAM, 2011). En el ámbito académico se han realizado estudios y textos en este sentido (Muñoz, 2011; Cheskin, Roberts y Margolis, 2012; Muñoz, 2012). 


\subsection{Papel de la escuela en la promoción del estilo de vida saludable}

La educación para la salud se basa en una propuesta pedagógica sustentada en contenidos científicos. En el caso de los libros de texto estos contenidos se tratan prioritariamente en Ciencias Naturales y en la colección Los libros del rincón, que se distribuyen en las bibliotecas escolares. Pero ya que el conocimiento científico no es acabado, los contenidos de los libros deben incluir aquellas aportaciones recientes que permitan considerarlas como avances conceptuales. Los mensajes que se emitan en los libros de texto han de ser "claros" (GF, 2010: 8; Igartua, 2011) y con la menor cantidad de errores y sesgos.

Sin embargo, cuestiones ideológicas pueden tergiversar las opiniones científicas para optar por posiciones menos conflictivas con estructuras de poder como sucede con los conceptos acerca de reproducción y sida (Hernández, Flores y Echeverría, 2011). En este marco es importante evaluar cuáles de estas ideas permean en los planes, programas, materiales impresos y en el diseño de las actividades que se encuentran vigentes en el sistema educativo de México.

Los libros de texto gratuitos de la escuela primaria de México fueron modificados para su publicación en 2011 de acuerdo a una reforma curricular impulsada por la Secretaría de Educación. El libro de sexto grado Ciencias Naturales, primero que se elaboró de acuerdo a los lineamientos pedagógicos de la reforma, no contiene temas ni propone actividades relacionados con la alimentación. Aunque Guerra y López (2011) no analizaron la validez de los contenidos de este volumen con base en la evidencia científica sino en las actividades descritas en el mismo, encontraron que se centran en la búsqueda de información y no en la elaboración de proyectos de ciencia. Además, las actividades carecen de coherencia interna.

Ese énfasis en la acumulación de información, desde la cual no se puede realizar un proceso de construcción de conocimiento, sustenta los materiales propuestos de Salazar et al. (2012) para su empleo en las escuelas primarias. Sin describir un análisis de los contenidos o los mensajes, elaboraron materiales educativos con los mismos ejes que se encuentran en los libros de texto gratuitos y el esquema del "Plato del buen comer". También se evalúan (desde la confusión entre evaluación y calificación) con exámenes de tipo test, lo que ya debería haber sido superado (Avilés, 2012) por lo que no contribuyen a formar a los educandos. El esquema del "Plato" que sustituyó a la "Pirámide de la Alimentación Ideal" de los libros elaborados en 1998, mantiene una postura simplista acerca de la alimentación saludable: es suficiente con un alimento de cada uno de las tres áreas del "Plato".

Estas visiones reduccionistas, con planteamientos pedagógicos hacia la transmisión del conocimiento, se han encontrado en textos escolares elaborados en otros países. La visión de la salud "como una serie de prohibiciones" (Gavidia, 2003) fue sustituida por la de la "dieta equilibrada" en las versiones posteriores de los libros escolares españoles (Barrio et al., 2008), también con el enfoque en equivalencia de alimentos por sus contenidos de macronutrimentos. Por otra parte, las imágenes de esos libros, que también pueden ser consideradas contenidos textuales, presentan "ausencia de información relevante" y "errores" conceptuales (Pérez et al., 1999). 


\section{Material y método}

El objetivo de este estudio fue analizar las características técnicas y el sustento científico de los mensajes relacionados con la nutrición en los libros de los educandos del nivel de educación primaria de México, con la hipótesis de que los contenidos de los libros de texto de la educación primaria no incorporan la evidencia científica. En este estudio fueron planteadas las siguientes preguntas de investigación:

1. ¿Cuáles son los ejes de los mensajes relacionados con la nutrición en los libros de texto gratuito?

2. ¿Los contenidos científicos que sustentan los mensajes en los libros de texto gratuito son pertinentes para sustentar las actividades escolares para la construcción del estilo de vida saludable?

3. ¿Los contenidos científicos de nutrición de los libros de las bibliotecas escolares son pertinentes para la realización de sus proyectos de aula?

Para responder a las preguntas de investigación se realizó un estudio observacional, analítico, de los mensajes acerca de alimentación de los libros de texto gratuitos de las escuelas primarias de México. El análisis de los mensajes se realizó por medio de un proceso de codificación del universo conformado por los contenidos relativos a salud en los libros de texto de los educandos, de segundo a sexto grado.

Se revisaron los libros que se emplean en el ciclo escolar agosto 2011-junio 2012, recuperados del sitio de la Reforma Integral de la Educación Básica de la Secretaría de Educación Pública de México (SEP, 2011), con primera edición en 2009. Se compararon con un ejemplar del quinto grado del ciclo 1999-2000. Así mismo, se analizaron las sugerencias para el maestro para el uso de libros de las bibliotecas escolares en la "Guía articuladora de materiales", disponible en el mismo sitio. Los libros forman parte de una colección que se distribuye en las escuelas: Los libros del rincón.

La variable independiente fue la existencia de mensajes acerca de la alimentación. Las variables dependientes fueron dos. Primero, si los mensajes se ajustan a las características técnicas de un buen mensaje: 1) deben ser claros, no ambiguos; 2) asociar los mensajes a sentimientos positivos; 3) deben motivar para el logro de metas (Igartua, 2011). Segundo, si los contenidos incorporaban los avances científicos (Muñoz, 2012).

Las unidades de análisis fueron los contenidos que se caracterizaron en tres categorías: 1) propósito o intencionalidad, cuyas subcategorías fueron nutrición saludable, consumo para la sustententabilidad, seguridad alimentaria; 2) características de los grupos de alimentos, donde las subcategorías fueron macronutrimentos y componentes bioactivos; 3 ) recomendaciones de alimentos con base en los efectos metabólicos y las subcategorías los que deben consumirse más, los que deben limitarse y los que deben evitarse ya que son obesogénicos y por tanto no son saludables, así como formas tradicionales de preparación.

Para la identificación de las oraciones clave se diseñó una matriz de captura. 


\section{Resultados}

Se encontró que la frecuencia más alta de contenidos sobre nutrición estuvo en el libro Ciencias Naturales de quinto grado del año 2011, 28 mensajes, que contrastan con los 11 del libro de 1999 (tabla 1). Éste último libro se publicó posteriormente a la Encuesta Nacional de Salud de 1996. En esa encuesta se encontró que el principal problema alimentario era el bajo peso en el $10 \%$ de los escolares. Eso contrastó con el aumento súbito de sobrepeso y obesidad en niños que se halló en la Encuesta Nacional de Salud y Alimentación de 2006, 26\% en conjunto.

Tabla 1. Frecuencias de los mensajes en los libros de los educandos

\begin{tabular}{|c|c|c|c|c|c|c|}
\hline & \multirow{3}{*}{$\begin{array}{r}\text { Inicio del curso } \\
\text { Grado escolar }\end{array}$} & \multicolumn{5}{|c|}{ Frecuencias } \\
\hline & & \multirow{2}{*}{$\frac{1998}{5^{\circ}}$} & \multicolumn{4}{|c|}{2011} \\
\hline & & & $3^{\circ}$ & $4^{\circ}$ & $5^{\circ}$ & $6^{\circ}$ \\
\hline Categorías & Subcategorías & & & & & \\
\hline \multirow[t]{5}{*}{ Propósitos explícitos } & 1.1 Bienestar & 1 & - & - & - & - \\
\hline & 1.2 Estilo vida saludable & 1 & - & 1 & 1 & 1 \\
\hline & 1.3 Nutrición saludable & 3 & 3 & - & 1 & - \\
\hline & 1.4 Seguridad alimentaria & 3 & 3 & - & - & - \\
\hline & 1.5 Enfermedades & - & - & 1 & 5 & - \\
\hline \multirow[t]{2}{*}{ Componentes } & 2.1 Nutrimentos & 1 & 4 & 1 & 1 & - \\
\hline & 2.2 Bioactividad & - & - & - & - & - \\
\hline \multirow{6}{*}{$\begin{array}{l}\text { Recomenda-ciones } \\
\text { para el consumo } \\
\text { saludable }\end{array}$} & 3.1 Consumirse más & - & 1 & 1 & 4 & - \\
\hline & 3.2 Consumirse menos & 1 & 2 & 1 & 5 & - \\
\hline & 3.3 Evitarse & - & - & 1 & 2 & - \\
\hline & 3.4 Equivalentes & 1 & 4 & 2 & 7 & - \\
\hline & 3.5 Tradiciones & - & - & 1 & 2 & - \\
\hline & Totales & 11 & 17 & 9 & 28 & 1 \\
\hline
\end{tabular}

Fuente: Contenido de los libros de texto gratuitos, Secretaría de Educación Pública de México, 2011

Los datos de la encuesta de 2006 explican el cambio en el énfasis que se encontró en el libro de $5^{\circ}$ grado de 2011, con 5 mensajes en referencia a enfermedades como obesidad, diabetes mellitus de tipo 2, enfermedad cardiovascular y artropatía por sobrepeso, lo que hizo crear otra subcategoría en la categoría propósitos explícitos. Excepto dos, los mensajes se localizaron en las secciones "La dieta correcta" y "Acciones para promover la salud". Las excepciones fueron la necesidad de desinfectar verduras y frutas, y que "una lata de refresco contiene 13 cucharaditas de azúcar" en el apartado correspondiente a química. 
El enfoque tradicional de la nutrición basado en los nutrimentos (carbohidratos, grasas y proteínas) es claro en las recomendaciones de alimentos y bebidas que han de consumirse más, menos o evitarse. Este enfoque, que sustenta las equivalencias de alimentos, cuya subcategoría es la que más veces se identificó, siete para $5^{\circ}$ grado, se refiere al esquema de la clasificación de alimentos en México, "El plato del buen comer", y un esquema para las bebidas, "La jarra del buen beber".

El siguiente libro en número de subcategorías identificadas fue el de $3^{\circ}$ grado, con 17 (tabla 1). Las dos secciones del libro, "Alimentación como parte de la nutrición" y "Dieta: los grupos de alimentos", sólo presentaron ocho de los mensajes. Nueve se encuentran como preguntas guías de los proyectos "Sobrepeso y obesidad son factores contrarios a la salud" y "Plato del buen comer y jarra del buen beber". Las preguntas como "productos naturales que se producen en el lugar donde viven", "alimentos de la región y de temporada" se consideraron en la subcategoría seguridad alimentaria pues, aunque no se relacionen en el texto, otro de los mensajes es el huerto escolar. El libro de $4^{\circ}$ grado tiene sólo nueve mensajes, entre ellos consumir hígado, pescado, cereales y leguminosas por el aporte de vitaminas del complejo B, dos veces "alimentarse correctamente" para evitar el sobrepeso y la obesidad, y "la manera de preparar alimentos es peculiaridad de cada país".

En las "Guía de materiales para ciencias naturales", ciclo 2011-2012 (tabla 2), se recomiendan ocho libros de la colección Los libros del Rincón, como apoyo al desarrollo de actividades. El eje de los mensajes de estos libros de apoyo es la identificación de los grupos de alimentos con base en macronutrimentos (carbohidratos, grasas, proteínas), lo cual se reitera, ya que las actividades pasivas (identificar, reconocer, comparar) para las cuales se sugiere la lectura de los mismos consideran la representación gráfica "El Plato del Buen Comer" como referente. Los libros de apoyo a las actividades escolares acerca de la alimentación fueron elaborados, por ejemplo, por autores de Estados Unidos (Alastair Smith, Kate Knigton, Ronne Randall), Cuba (Olimpia Carrillo Farnés), España (Josep Comellas Humet), México (Patricia Wriedt). Esta diversidad de países de origen de los autores muestra la persistencia de una visión tradicional global de las recomendaciones acerca de la alimentación, donde el centro son los grupos de alimentos y las equivalencias. 
Tabla 2. Sugerencias para apoyar la planeación didáctica y libros de consulta

\begin{tabular}{|c|c|}
\hline Sugerencias & Libros \\
\hline \multicolumn{2}{|c|}{$2^{\circ}$ grado } \\
\hline $\begin{array}{l}\text { Analiza su alimentación con base en los tres } \\
\text { grupos de alimentos y sus horarios de comida. }\end{array}$ & $\begin{array}{l}\text { Randall, R. (2007). ¿De dónde provienen los } \\
\text { alimentos que hay en tu refrigerador? }\end{array}$ \\
\hline \multicolumn{2}{|c|}{$3^{\circ}$ grado } \\
\hline $\begin{array}{l}\text { Identifica la importancia de la nutrición en el } \\
\text { crecimiento y buen funcionamiento del cuerpo. }\end{array}$ & $\begin{array}{l}\text { Randall, R (2005). ¿Qué tienen de bueno las } \\
\text { verduras? }\end{array}$ \\
\hline Reconoce el aporte nutrimental de los alimentos & Baumbush, B. (2002). Comida. \\
\hline $\begin{array}{l}\text { de cada grupo representados en "El plato del } \\
\text { buen comer" relacionándolos con los que se } \\
\text { producen en su comunidad. }\end{array}$ & Wriedt, P. (2005). No por mucho masticar... \\
\hline $\begin{array}{l}\text { Compara los alimentos que consume } \\
\text { regularmente con "El plato del buen comer" en } \\
\text { términos de una dieta equilibrada. }\end{array}$ & $\begin{array}{l}\text { Arriaga, A; Osornio, A. (2005). Lo que hago } \\
\text { con mi cuerpo. }\end{array}$ \\
\hline \multicolumn{2}{|c|}{$4^{\circ}$ grado, bloque 5 , ámbito 1} \\
\hline $\begin{array}{l}\text { Relaciona las funciones del cuerpo humano con } \\
\text { el mantenimiento de la salud. }\end{array}$ & Smith, A. (200 \\
\hline \multirow{4}{*}{$\begin{array}{l}\text { Explica la importancia de fomentar y poner en } \\
\text { práctica hábitos que promuevan la salud } \\
\text { personal. }\end{array}$} & Smith, A. (2003). ¿Qué pasa cuando comes? \\
\hline & $(2005)$ I0 \\
\hline & $\begin{array}{l}\text { con mi cuerpo. } \\
\text { col } A \text {. }\end{array}$ \\
\hline & Carrillo Farnes, O. (2005). Nutrición y salud. \\
\hline \multicolumn{2}{|c|}{$5^{\circ}$ grado, bloque 5 , ámbito 1} \\
\hline $\begin{array}{l}\text { Explica la importancia de fomentar y poner en } \\
\text { práctica hábitos que promueven la salud } \\
\text { personal. }\end{array}$ & $\begin{array}{l}\text { Arriaga, A; Osornio, A. (2005). Lo que hago } \\
\text { con mi cuerpo. México: SEP-Santillana. } \\
\text { Smith, A. (2003). ¿Qué pasa cuándo comes? } \\
\text { Carrillo Farnes, O. (2005). Nutrición y salud. }\end{array}$ \\
\hline \multicolumn{2}{|c|}{$5^{\circ}$ grado, bloque 5 , ámbito 1} \\
\hline \multirow{2}{*}{$\begin{array}{l}\text { Explica las caracteristicas de una dieta correcta } \\
\text { en relación con sus necesidades nutrimentales y } \\
\text { las costumbres alimentarias de la comunidad. } \\
\text { Describe las causas y consecuencias de algunos } \\
\text { problemas de la alimentación y reconoce la } \\
\text { importancia de mejorar los hábitos alimentarios. }\end{array}$} & Carrillo Farnes, O. (2005). Nutrición y salud. \\
\hline & $\begin{array}{l}\text { Carrillo Farnes, O. (2005). Nutrición y salud. } \\
\text { Knigton, K. (2005). ¿Comida basura? No } \\
\text { gracias. }\end{array}$ \\
\hline \multicolumn{2}{|c|}{$6^{\circ}$ grado, bloque 5 , ámbito 1} \\
\hline $\begin{array}{l}\text { Argumenta la importancia de la dieta para } \\
\text { fortalecer el funcionamiento del sistema } \\
\text { inmunológico. }\end{array}$ & $\begin{array}{l}\text { Comellas Humet, J. (2005). Hábitos inteligentes } \\
\text { para tu salud. } \\
\text { Carrillo Farnes, O. (2005). Nutrición y salud. }\end{array}$ \\
\hline
\end{tabular}

Fuente: Guía de materiales para ciencias naturales, Secretaría de Educación Pública, 2011

En la percepción de la nutrición con este enfoque reduccionista, con tareas diseñadas para la escuela tradicional más que para la construcción de competencias (hacer-saber hacer), se pasa por alto que la ciencia es tanto una construcción social como un cuerpo de conocimiento en constante construcción. De esta manera se omite que la educación científica no sólo debe incorporar avances conceptuales e instrumentales (los cuales no se encuentran en los mensajes de los libros escolares analizados) sino también valorar la educación acerca de la ciencia como parte de las prácticas escolares (lo cual no es posible con tareas pasivas y no tendientes a una educación para la vida, de acuerdo a la revisión de las sugerencias a la planeación didáctica).

\section{Discusión}

Se realizó esta investigación para analizar la validez de los contenidos de los mensajes acerca de la alimentación en los Libros de Texto Gratuitos de la escuela primaria de México. En estudios 
anteriores se encontraron errores acerca de anatomía y fisiología (Pérez et al., 1999), se han explicitado inconsistencias en la lógica interna de la construcción de los textos (Gavidia, 2003; García y Martínez, 2005; Barrio, 2008; Flores et al., 2008), y se ha polemizado de la conveniencia de medicalizar (Barrio et al., 2008) o no (Gavidia, 2003) los contenidos. Además de revisar las características de los mensajes de los libros, nuestro estudio analiza si los conceptos se basan en la evidencia científica.

\subsection{Dificultades por la estructura de los mensajes}

El libro de texto debe ser atractivo para los lectores para lograr un contrapeso a los mensajes que los educandos miran cotidianamente en los medios. Como mensajes para formar un estilo de vida saludable presentan dos grandes dificultades. La presentación de los contenidos no sigue los lineamientos deseables para elaborar mensajes; las recomendaciones se centran en las equivalencias de los alimentos y en los macronutrimentos. Estas dos características amplias las comparten con los libros de apoyo (tabla 2). De acuerdo con las recomendaciones para la creación de una "publicidad trascendente" los mensajes deben "enganchar" (Igartua, 2011) y para ello han de ser directos, sin ambigüedades, emplear atractivos psicológicos y promover mensajes positivos encaminados a alentar un consumo.

\subsubsection{Los mensajes han de ser claros}

En el libro de $5^{\circ}$ grado los mensajes sobre el riesgo de padecer una enfermedad crónica no transmisible no se sustentaron en las recomendaciones para el consumo saludable. Si bien se encontraron cinco de alimentos y bebidas que deben "consumirse menos" (tabla 1), no fueron mensajes que resultaran atractivos; antes bien, resultan ambiguos. Por ejemplo, no se define lo que significa "alimentación correcta". No se logra la asociación que debería establecerse entre la salud como "estado de bienestar". A pesar de la evidencia científica que atribuye a las bebidas azucaradas con o sin gas un papel relevante en la pandemia de sobrepeso y obesidad, sólo se menciona de manera ambigua que "el refresco podría ser una de las causas del problema de la obesidad". Así, no se hace atractivo el consumo de agua simple potable. Aunque está clara la asociación en el consumo de alimentos obesogénicos con la pandemia, se encontró una recomendación tibia: "evitar el consumo excesivo de alimentos industrializados, refrescos, frituras".

\subsubsection{Falta asociar la alimentación correcta a sentimientos positivos}

En el libro de $5^{\circ}$ grado de 1999 se encontró un mensaje en este sentido. Esto ya no está en el de 2011 , sino un mensaje discriminatorio. En la página 19 se encuentra una tira cómica en la cual se culpa a una niña obesa de serlo por comer en la escuela, "a veces tomamos refrigerios", con lo que culpa a la niña de su comportamiento como si ella no viviera en un contexto social del cual recibe esta influencia. Esta negatividad (o medicalización) se encuentra en que la frecuencia mayor correspondió a enfermedades y alimentos y bebidas que deben consumirse menos, 12 de 28 subcategorías (tabla 1). Este énfasis negativo se encuentra en el título de uno de los libros de consulta; el de Knighton se llama ¿Comida basura?, no gracias (2005). Esto no considera uno de los más importantes elementos de los mensajes comerciales, la asociación de los 
mismos a situaciones de sentimiento de valor social, independencia, poder y éxito; por ejemplo se destapa felicidad, no una soda, tal como afirma la mercadotecnia (Coca Cola México). Por consiguiente en la escuela no se asocia el comer al disfrute de alimentos saludables y poco procesados.

\subsubsection{El énfasis de los mensajes}

En los procesos escolares el énfasis de los mensajes debe ser la motivación para el logro de la salud (Gavidia, 2003; Igartua, 2011). Éste disminuyó en las nuevas ediciones de los libros de texto. En el libro de $5^{\circ}$ grado de 1999 fueron ocho (72\% de los relativos a la alimentación) los mensajes en la categoría de propósitos explícitos hacia este objetivo (bienestar, estilo de vida y nutrición saludables); sólo dos $(7 \%)$ en el de $5^{\circ}$ grado y siete en total $(13 \%)$ en los libros de $3^{\circ}$ a $6^{\circ}$ grado en las ediciones de 2011 (tabla 1).

\subsection{Dificultades por los contenidos de los mensajes}

Por otra parte, el contenido del mensaje debe cambiarse. Los enfoques, tanto en los libros de los educandos como en los materiales de apoyo para los proyectos de aula, han dejado fuera los avances científicos que sustentan la necesidad de la reconfiguración de los enfoques de la nutrición. La idea de una dieta balanceada y las equivalencias (tabla 1), presenta como semejantes la papa (un tubérculo), harinas refinadas y cereales integrales. La idea de las equivalencias domina el contenido de los mensajes, $24 \%$ de los que se identificaron en los libros de $3^{\circ}$ a $5^{\circ}$ grado en la edición de 2011. Esto no permite que los educandos aprecien el valor de los alimentos poco procesados y la necesidad de alimentarse con base en alimentos específicos (Mozaffarian, Hao, Rimm, Willet y Hu, 2011; Willet y Ludwig, 2012) como panes de trigo integral, papillas de avena o tortillas de maíz (Fardet, 2010).

En el libro de Comellas (tabla 2) se recomiendan productos escasamente saludables: la mermelada, incluso la reducida en azúcar, tiene demasiada fructosa, está asociada a cirrosis hepática no alcohólica (Nomura y Yamanouchi, 2012), que es componente principal de la quinta causa de muerte general en México; los cereales de caja para el desayuno no son integrales y tienen hasta el $30 \%$ de azúcar extra (PROFECO, 2011); el jamón, como todos los productos de carne procesada, así sea de pavo, se asocia a riesgo de algunos tipos de cáncer a causa de los nitratos (WCRF, 2006) y "no debe ser recomendado por los nutricionistas" (Aguilar, 2010).

Este énfasis tampoco considera características de la percepción de los niños. Para ellos "las cosas no son parte de una cadena de montaje, no ocupan un lugar en los recursos funcionales de un sistema, cada cosa adquiere significación propia" (Benjamin, 1988). Cambiar el enfoque para transitar hacia la recomendación con base en alimentos específicos y no una clasificación, puede darle sentido al educando a esas recomendaciones desde una postura simplificante, pero no simple, ya que considera la complejidad de la interacción de los constituyentes de los alimentos. De esta manera el aprendizaje esperado a partir de los textos, actividades y literatura de apoyo de estos libros de ciencias, es pobre (Guerra et al., 2011). Peor, porque si se lograra el aprendizaje significativo se haría a partir de errores conceptuales. Así, la educación no se configura hacia el logro de la capacidad de los educandos para transitar hacia modos saludables 
de consumo de alimentos.

\section{Conclusión}

El estudio no se dirigió hacia la percepción que tienen los educandos, sus maestros y las familias acerca de los mensajes. Ésta es una limitante para la elaboración de conclusiones. Sin embargo, se encontró que las recomendaciones analizadas en los libros escolares para la correcta nutrición no consideran la complejidad de los alimentos y sus efectos en el organismo.

Las prácticas escolares no han mostrado ser efectivas para disminuir el aumento del número de personas que cada año padecen algún tipo de trastorno a causa del consumo de los alimentos y bebidas poco saludables ni para contrarrestar el efecto de la mercadotecnia, ya que no hay estudios consistentes que demuestren que a mayor escolaridad menor sobrepeso y obesidad.

Es importante que la educación para una nutrición saludable incorpore los avances científicos pero también considere los saberes ancestrales, reconfigure el valor cultural de la cocina tradicional y se recuperen las prácticas alimenticias que fortalecen la salud como posibilidad para un desarrollo sustentable.

Con los datos actuales es posible elaborar recomendaciones de base para sustentar un estilo de alimentación, sin que sea impositiva, considerando el contexto en los cuales se desarrollan las poblaciones y las posibilidades de recuperación de saberes culinarios ancestrales para cada comunidad con las modificaciones que le den mayor variedad y riqueza.

Es necesario reconfigurar el paradigma en que se sustentan las recomendaciones tradicionales de la nutrición e incorporar los hallazgos científicos para conformar una nutrición basada en la evidencia que a su vez legitime la alimentación ancestral, local, producto de milenios de ensayo y error. Las aplicaciones de la ciencia deberán canalizarse al logro de un estado óptimo de salud y no en forma de restricciones dietéticas para resolver problemas de desnutrición, sobrepeso y obesidad. Las actividades escolares tendentes a fomentar una educación para el estilo de vida saludable, al sustentarse en textos creados bajo el enfoque de la clasificación simple de los alimentos con base en macronutrimentos, no cuentan con bases sólidas para hacerlo. Los profesionales de la salud deberían hacer las recomendaciones para educar en la selección de alimentos más saludables. Esto debe reflejarse en los mensajes dirigidos a los educandos de las escuelas primarias en sus libros de texto.

\section{Referencias}

Aguilar, Armando. (2010). Salchichas para hot dog. Revista del Consumidor. N ${ }^{\circ} 34$, pp. 34-45. Disponible en http://revistadelconsumidor.gob.mx/wp-content/uploads/2010/10/salchichas2.pdf. Recuperado el 4 de enero de 2013. 
Anzures, Tonatiuh. (2011). El libro de texto gratuito en la actualidad. Logros y retos de un programa cincuentenario. Revista Mexicana de Investigación Educativa. Vol. 49, n 16, pp. 363388. Disponible en

http://www.seg.guanajuato.gob.mx/CEducativa/Documents/2011/CentroDocumental/Mayo/130 52011/El\%20libro\%20de\%20texto\%20gratuito.pdf. Recuperado el 3 de enero de 2013.

Avilés, Karina. (2012). Cuestiona la OCDE enfoque dado a evaluación de la enseñanza. La Jornada en Línea. Disponible en

http://www.jornada.unam.mx/2012/11/09/sociedad/040n1soc?partner=rss. Recuperado el 3 de enero de 2013.

Barrio Cantalejo, Inés M.; Ayudarte Larios, M. Luisa; Hernán García, Mariano et al. (2008). Presencia de las actuales prioridades de salud infantil y adolescente en los libros de texto escolares. Gaceta Sanitaria. Vol. 3, $\mathrm{n}^{\circ}$ 22, pp. 227-231. Disponible en http://scielo.isciii.es/pdf/gs/v22n3/breve1.pdf. Recuperado el 3 de enero de 2013.

Benjamin, Walter. (1970). Sobre algunos temas en Baudeliere. En Sobre el programa de la filosofía futura y otros ensayos. Caracas: Monte Ávila.

Cheskin, Lawrence J.; Roberts, Carmen y Margolis, Simeon. (2012). The 2012 Johns Hopkins Nutrition and Weight Control White Paper. Disponible en

http://www.johnshopkinshealthalerts.com/white_papers/nutrition_weight_control_wp/D_landing. html. Recuperado el 3 de enero de 2013.

Coca Cola México. (s.f.). Destapa la felicidad. Disponible en http://www.cocacola.com.mx/es/index.html\#. Recuperado el 3 de enero de 2013.

De los Reyes, Ignacio. (2011). Comida mexicana, la esperanza de salvar al país de la obesidad. BBC Mundo. Disponible en

http://www.bbc.co.uk/mundo/noticias/2011/08/110802_mexico_obesidad_comida_esperanza_ir m.shtml. Recuperado el 3 de enero de 2013.

Fardet, Anthony. (2010). New Hypotheses for the Health-Protective Mechanisms of Whole-Grain Cereals: What is Beyond Fibre? Nutrition Research Reviews. $N^{\circ}$ 23, pp. 65-134. doi: $10.1017 /$ S0954422410000041

Flores Huerta, Samuel; Klünder Klünder, Miguel y Medina Bravo, Patricia. (2008). La escuela primaria como ámbito de oportunidad para prevenir el sobrepeso y la obesidad en los niños. Boletín Médico del Hospital Infantil de México. $\mathrm{N}^{\circ}$ 65, pp. 626-638. Disponible en http://www.medigraphic.com/pdfs/bmhim/hi-2008/hi086r.pdf. Recuperado el 3 de enero de 2013.

García Barros, Susana y Martínez Losada, Cristina. (2005). La nutrición en textos escolares del último ciclo de primaria y primero de secundaria. Enseñanza de las Ciencias. $\mathrm{N}^{\circ}$ extra. Disponible en http://www.uruguayeduca.edu.uy/Userfiles/P0001/File/Garcia_Barros_028[1].pdf. Recuperado el 3 de enero de 2013. 
Gavidia Catalán, Valentín. (2003). La educación para la salud en los manuales escolares españoles. Revista Española de Salud Pública. Vol. 77, n² 2, pp. 275-285. Disponible en http://scielo.isciii.es/pdf/resp/v77n2/original9.pdf. Recuperado el 4 de enero de 2013.

Gobierno Federal de México. (2010). Programa de acción en el contexto escolar: lineamientos básicos para el expendio o distribución de alimentos y bebidas en los establecimientos de educación básica. Acuerdo Nacional para la Salud Alimentaria. Estrategia contra el sobrepeso y la obesidad. Disponible en

http://portal.salud.gob.mx/sites/salud/descargas/pdf/salud_alimentaria/programa_accion.pdf.

Recuperado el 3 de enero de 2013.

Guerra Ramos, María Teresa y López Valentín, Dulce María. (2011). Las actividades incluidas en el libro de texto para la enseñanza de las ciencias naturales en sexto grado de primaria. Análisis de objetivos, procedimientos y potencial para promover el aprendizaje. Revista Mexicana de Investigación Educativa. Vol. 49, n 16, pp. 441-470. Disponible en http://scielo.unam.mx/pdf/rmie/v16n49/v16n49a6.pdf. Recuperado el 3 de enero de 2013.

Hernández Rosete, Daniel; Flores, Javier y Echavarría, Laura. (2011). Sin pecado concebido. Sida y embarazo en el libro de sexto de ciencias naturales. Revista Mexicana de Investigación Educativa. Vol. 49, n 16, pp. 471-488. Disponible en http://www.scielo.org.mx/pdf/rmie/v16n49/v16n49a7.pdf. Recuperado el 3 de enero de 2013.

Igartua, Juan José. (2011). Mejor convencer entreteniendo: comunicación para la salud y persuasión narrativa. Revista de Comunicación y Salud. Vol. 1, n 1, pp. 69-83. Disponible en http://www.revistadecomunicacionysalud.org/index.php/rcys/article/view/12/7. Recuperado el 3 de enero de 2013.

Mozaffarian, Dariush; Hao, Tao; Rimm, Eric B.; Willet, Walter C. y Hu Frank B. (2011). Changes in Diet and Lifestyle and Long-Term Weight Gain in Women and Men. New England Journal of Medicine. $\quad \mathrm{N}^{\circ}$ 364, pp. 2.392-2.404. Disponible en http://www.foodpolitics.com/wpcontent/uploads/NEJMoa1014296.pdf. Recuperado el 3 de enero de 2013.

Muñoz Cano, Juan Manuel. (2011). Dieta tradicional tabasqueña. Componentes bioactivos, alimentos funcionales. Disponible en http://www.archivos.ujat.mx/DACS/publicaciones/publicaciones_dacs/produccion_investigador es/3_Libro-Componentes-bioactivos-alimentos-funcionales.pdf. Recuperado el $\overline{3}$ de enero de 2013.

Muñoz Cano, Juan Manuel. (2012). Alimentación humana. De la experiencia tradicional a la evidencia científica. Disponible en

http://www.archivos.ujat.mx/DACS/publicaciones/publicaciones_dacs/produccion_investigador es/4-Libro-alimentacion-humana.pdf. Recuperado el 3 de enero de 2013.

Nakamura, Yukiko K. y Omalle, Stanley T. (2012). Metabolic Diseases and Pro- and Prebiotics. Mechanistic Insights. Nutrition \& Metabolism. Vol. 60, n 9. doi:10.1186/1743-7075-9-60 
Nomura, Kyoko y Yamanouchi, Toshikazu. (2012). The Role of Fructose-Enriched Diets in Mechanisms of Nonalcoholic Fatty Liver Disease. The Journal of Nutritional Biochemistry. Vol. 3, $n^{\circ}$ 23, pp. 203-208. doi:10.1016/j.jnutbio.2011.09.006

Pérez de Eulate, Lourdes; Llorente, Enrique y Andrieu, Amaya. (1999). Las imágenes de digestión y excreción en los textos de primaria. Enseñanza de las Ciencias. Vol. 2, $\mathrm{n}^{\circ} 17$, pp. 165-178. Disponible en http://ddd.uab.es/pub/edlc/02124521v17n2p165.pdf. Recuperado el 3 de enero de 2013.

Procuraduría Federal del Consumidor. (2011). Cereales para niños ¿Sabes cuánta azúcar consumen? La otra cara del inocente desayuno. Revista del Consumidor. N 41, pp. 30-44.

Salazar Coronel, Araceli; Shamah Levy, Teresa; Escalante Izeta, Ericka I. y Jiménez Aguilar, Alejandra. (2012). Validación de material educativo: estrategia sobre alimentación y actividad física en escuelas mexicanas. Revista Española de Comunicación en Salud. Vol. 2, n 3, pp. 96109. Disponible en http://www.aecs.es/3_2_2.pdf. Recuperado el 3 de enero de 2013.

Secretaría de Educación Pública de México. (2011). Reforma Integral. Disponible en http://basica.sep.gob.mx/reformaintegral/sitio/index.php?act=frontlibros. Recuperado el 3 de enero de 2013.

UNESCO. (2010). La cocina tradicional mexicana, cultura comunitaria, ancestral y viva. El paradigma de Michoacán. Disponible en

http://www.unesco.org/culture/ich/index.php?lg=es\&pg=00011\&RL=00400. Recuperado el 3 de enero de 2013.

United States Departament of Agriculture. (2011). Choose MyPlate. Disponible en http://www.choosemyplate.gov/. Recuperado el 3 de enero de 2013.

Universidad Autónoma de México (UNAM). (2011). La cocina tradicional mexicana, una respuesta al sobrepeso y obesidad. México: Boletín DGCS-610 de la UNAM. Disponible en http://www.dgcs.unam.mx/boletin/bdboletin/2011_610.html. Recuperado el 4 de enero de 2013.

Willet, Walter C. y Ludwig, David S. (2012). The 2010 Dietary Guidelines - The Best Recipe for Health? New England Journal of Medicine. Vol. 17, $\mathrm{n}^{\circ} 365$, pp. 1.563-1.565. Disponible en http://www.nejm.org/doi/pdf/10.1056/NEJMp1107075. Recuperado el 3 de enero de 2013.

World Cancer Reseach Fund, American Institute for Cancer Research. (2006). Food, Nutrition, Physical Activity, and the Prevention of Cancer: a Global Perspective. Disponible en http://www.dietandcancerreport.org/expert_report/recommendations/index.php. Recuperado el 3 de enero de 2013. 